\title{
Which factor is the most effective one in metabolic Sydrome on the outcomes after coronary artery bypass graft surgery? A cohort study of 5 Years
}

Lijuan Wang ${ }^{1+}$, Xiangyang Qian², Mingya Wang ${ }^{1}$, Xinran Tang ${ }^{1}$ and Hushan Ao ${ }^{1{ }^{*+}}$

\begin{abstract}
Background: Metabolic Syndrome and diabetes mellitus are risk factors for cardiovascular disease. However, the effects of Metabolic Syndrome with or without diabetes on perioperative and long-term morbidity and mortality after Coronary Artery Bypass Graft remain unclear.

Methods: An retrospective study was performed on 1166 patients who received isolated primary Coronary Artery Bypass Graft in Fuwai hospital. They were divided into three groups: control, Metabolic Syndrome of three factors together with diabetes and Metabolic Syndrome of three factors without diabetes ( $n=868,76$ and 222 respectively). Analysis of variance, Chi-rank test, Fisher's exact test, Log-rank test, Cox and Logistic regression models were used for data processing. Outcomes were postoperative and 5-year survival and morbidities.

Results: There were no significant differences between groups in in-hospital postoperative complications, epinephrine use, stroke, atrial fibrillation, renal failure, coma, myocardial infarction and repeated revascularization. Patients in the Metabolic Syndrome with diabetes, Metabolic Syndrome without diabetes and control groups, respectively, showed significant difference in perioperative Major Adverse Cerebral Cardiovascular Events (30.3\% vs. 21.2\%, 16.7\%, $P=0.0071$ ) and mortality (11.8\% vs. $2.7 \%, 3.11 \%, P=0.0003)$. The Metabolic Syndrome with diabetes group had higher rates of perioperative mortality than Metabolic Syndrome without diabetes $(P=0.0017, P$ of Fisher Test $=0.0039)$. Compared with non-diabetic patients with Metabolic Syndrome, those with Metabolic Syndrome and diabetes had increased long-term mortality (Adjusted HR: 4.3; 95\% Cl: 1.4-13.3; $P=0.0113$ ) and Major Adverse Cerebral Cardiovascular Events (Adjusted OR: 1.7; 95\% Cl: 1.0-2.8; $P=0.048$ ). Control and non-diabetic Metabolic Syndrome groups did not differ in long-term mortality but controls had lower rates of Major Adverse Cerebral Cardiovascular Events (Adjusted OR: 0.79; 95\% Cl: 0.64-0.98; $P=0.0329$ ).

Conclusions: There were significance differences between the three groups in perioperative Major Adverse Cerebral Cardiovascular Events and mortality after Coronary Artery Bypass Graft. Compared with non-diabetic Metabolic Syndrome patients, patients with Metabolic Syndrome and diabetes had higher long-term Major Adverse Cerebral Cardiovascular Events and mortality. While patients free of MetS and diabetes were associated with lower incidence of long-term Major Adverse Cerebral Cardiovascular Events after Coronary Artery Bypass Graft.
\end{abstract}

Keywords: Metabolic syndrome, Coronary artery bypass graft surgery, Diabetes, Major adverse cerebral cardiovascular events

\footnotetext{
* Correspondence: aohushan@126.com

${ }^{\dagger}$ Equal contributors

'Department of Anesthesiology, Beijing Fuwai Hospital, National Center for

Cardiovascular Diseases, Chinese Academy of Medical Sciences and Peking

Union Medical College, No. 167, Beilishi Road, West District of Beijing, Beijing

100037, China

Full list of author information is available at the end of the article
} 


\section{Background}

The incidence of Metabolic Syndrome (MetS) has been increasing worldwide due to changing lifestyles. According to Diagnosis and management of the metabolic syndrome: an American Heart Association; National Heart, Lung, and Blood Institute Scientific Statement, MetS can be diagnosed as the presence of any three of the following five criteria: increased body mass index, elevated triglycerides, reduced high-density lipoproteincholesterol, elevated blood pressure and elevated fasting glucose [1]. The incidence of MetS in patients who receive cardiovascular surgical procedures is quite high (nearly 46\%) [2] almost double the rate in the general population (23-28\%) [3]. Diabetes is also increasingly more prevalent among patients with Coronary Heart Disease (CHD) who need Coronary Artery Bypass Graft Surgery (CABG). Studies show that one-quarter to onehalf of MetS patients receiving CABG also suffer from diabetes $[2,4,5]$. Further, MetS is associated with higher mortality and morbidity after CABG [6, 7]. Diabetes is an independent risk factor for poor outcomes after cardiac surgery $[8,9]$. One study of 235 patients found diabetes and MetS were risk factors for prolonged ICU stays $(>5$ days) and atelectasia $(P<0.05)[10]$. There was also a significant associations between diabetes and pulmonary embolism $(P=0.025)$ and mediastinitis $(P=$ 0.051). However, in most studies patients with MetS may have diabetes. There are few long-term clinical studies that compared the outcomes of MetS patients with or without diabetes after CABG, especially in Chinese cohort. So it's not clear enough about which factor in MetS contributes mostly to the bad outcomes. The present study aimed to find out the impact of MetS with or without diabetes on 30-day and 5-year mortality and MACCE in patients undergoing CABG.

\section{Methods \\ Study design}

This study enrolled from 4916 consecutive Chinese patients who underwent isolated primary CABG at Fuwai Hospital in Beijing, China. The cases were collected from January 1, 1999 to December 30, 2005. All patient records and information were anonymized and at equal prior to analysis. Authors had no access to information that could identify individual participants during or after data collection. As shown in Fig. 1, among them 1166 patients met the inclusion criteria of this research, which was sorted at 2015 December. The inclusion criteria for the patients were as follows: (1) aged more than 18 years old, (2) had a definite history of CHD (CHD was defined as having a history of myocardiac ischemia, percutaneous intervention, thrombolytic therapy or a documented angiogram with visualized luminal obstruction or irregularity), (3) no severe illness of other systems, (4) received CABG surgery, (5) no trauma, infection, tumor, or previous surgery. The 1166 patients were divided into three groups: control $(n=868)$, MetS with diabetes $(n=$ 76) and non-diabetes MetS $(n=222)$. Controls were patients with no MetS criteria. The MetS with diabetes group included patients with diabetes and any 3 out of these 4 criteria: excess body mass index, hypertension, hypertriglyceridemia and low high-density lipoprotein

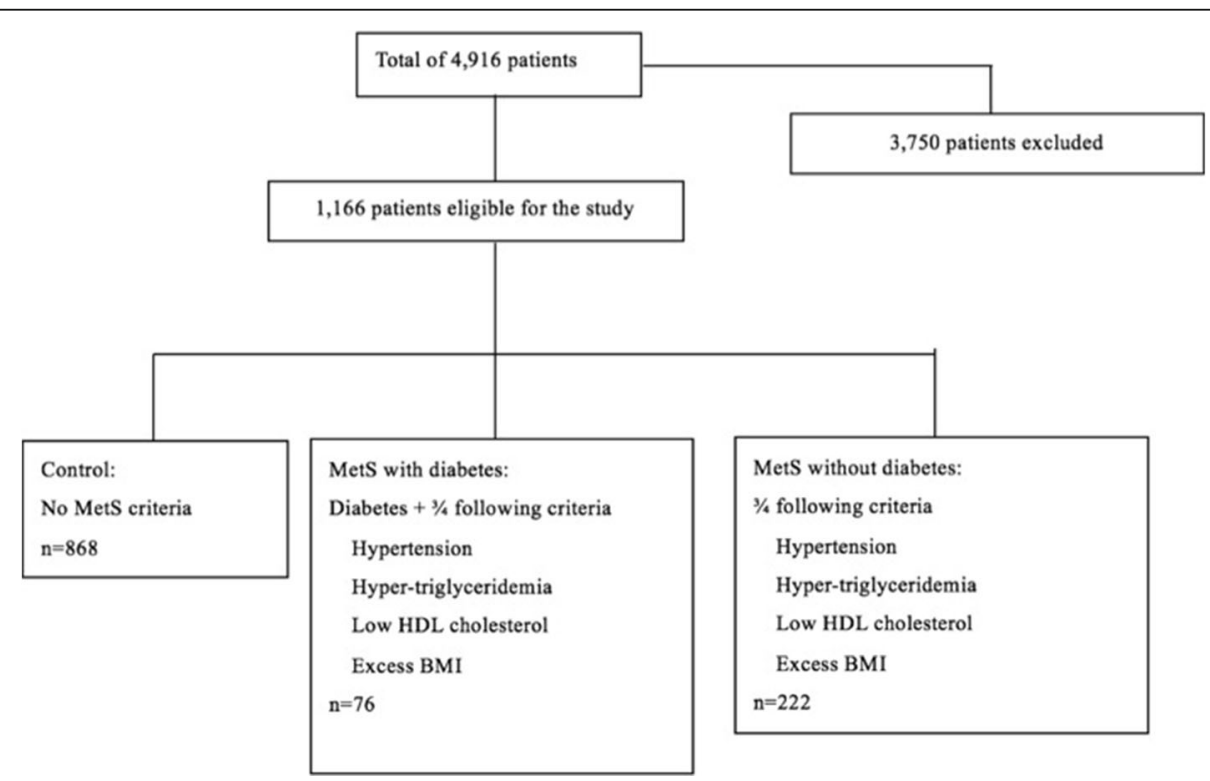

Fig. 1 Study population recruitment summary. This study enrolled from 4916 consecutive Chinese patients who underwent isolated primary CABG at Fuwai Hospital. Among them 1166 patients met the inclusion criteria of this research, who were divided into three groups: control $(n=868)$, MetS with diabetes $(n=76)$ and non-diabetes MetS $(n=222)$ 
cholesterol. The non-diabetes MetS group were patients with 3 of 4 criteria: excess body mass index, hypertension, hypertriglyceridemia and low high-density lipoprotein cholesterol with no hypertriglyceridemia or diabetes. The exact criteria of MetS can be found in the part of Identification of patients with MetS and diabetes. Data were obtained based on these conditions and were used to do statistical analysis.

\section{Operative techniques}

All patients received standardized anesthetic and surgical techniques, including access through a mid-sternotomy. After surgery, patients were transferred to the ICU and extubated as soon as they were stable: body temperature at normal level, awake, hemodynamic stability and no significant bleeding.

\section{Identification of patients with MetS and diabetes}

In this study, the clinical diagnosis of the MetS was made according to modified Adult Treatment Panel III of the National Cholesterol Education Program which requires meeting at least three criteria for diagnosis [11]. Obesity: body mass index greater than $28 \mathrm{~kg} / \mathrm{m}^{2}$. Hypertriglyceridemia: elevated triglycerides $(>150 \mathrm{mg} / \mathrm{dL}$ or drug treatment) or low high-density lipoprotein cholesterol: reduced high-density lipoprotein cholesterol $(<40 \mathrm{mg} / \mathrm{dL}$ in men, $<50 \mathrm{mg} / \mathrm{dL}$ in women, or drug treatment). Hypertension: elevated arterial blood pressure $(>130 \mathrm{mmHg}$ systolic, $>85 \mathrm{mmHg}$ diastolic, or drug treatment). And hyperglycemia: elevated fasting glucose (FPG $>100 \mathrm{mg} / \mathrm{dL}$ or drug treatment). The previous criterion to classify obesity in the Adult Treatment Panel III(2005) was waist circumference > $120 \mathrm{~cm}$ in men, $>88 \mathrm{~cm}$ in women, but this was not measured in this cohort. Therefore we used BMI instead of waist circumference. Recent studies showed that most MetS patients with excess BMI also have excess waist circumference [12, 13]. Diabetes mellitus (DM) was defined as a fasting plasma glucose level $\geq$ $126 \mathrm{mg} / \mathrm{dl}$ or requiring treatment with oral hypoglycemic medication or insulin use.

\section{Outcome events definition}

Renal failure was defined as progressive oliguria or anuria or need for dialysis [14]. Stroke was defined as central nervous systerm (CNS) dysfunction lasting more than $72 \mathrm{~h}$. Coma was defined as loss of consciousness for more than $24 \mathrm{~h}$. Outcomes that ended in death and MACCE were recorded from follow-up. MACCE was defined as permanent or transient stroke, coma, perioperative myocardial infarction (MI), heart block, and cardiac arrest [15-17].

\section{Data sources}

All these patients' basic characteristics, perioperative data, a short-term follow-up ( $\leq 30$ days after operation [18]) and a long-term follow-up (annually from the first year to the fifth year after surgery [19]) data were documented in Fuwai Hospital CABG case database. Basic characteristics and perioperative data including medical history, course in hospital, course during operation, examination and laboratory test and so on were from the electronic record of each patients. Patients' 30 days situation and annually situation was obtained by telephone follow-up and documented in Fuwai Hospital CABG case database as well.

\section{Statistical analysis}

Continuous variables were described in the form of mean \pm standard deviation and compared by analysis of variance (ANOVA). Categorical variables were treated as frequency and percentages, then compared by Chisquare test or Fisher's exact test. Kaplan-Meier curves represented 5-year cumulative mortalities and compared by log-rank test. Follow-up mortalities were analyzed by univariate Cox regression and multiple Cox regression models and MACCE by univariate and multiple Logistic regression models. According to previous literature, clinical observation and characteristics that showed significant difference among 3 groups in baseline variables were made to be covariates in the multiple regression models. Covariates included age, sex, smoking, aortic cross-clamp time, cerebrovascular events, peripheral artery disease, thrombolytic therapy, myocardial infarction and left main disease. All statistical tests were performed with the SAS 9.13 software (SAS Institute, Cary, NC, USA). The significance level was set at 0.05 and all tests were two-sided.

\section{Results}

Baseline and intraoperative characteristics

Of 4916 eligible patients in the database, 1166 patients met the inclusion criteria and were divided into three groups as shown in Fig. 1. Baseline data of the patients are presented in Table 1. No significant differences were found between the three groups in age, smoking, preoperative creatinine, cardiopulmonary bypass (CPB) time, aortic cross-clamp (ACC) time, diseased coronary artery, ejection fraction (EF) value on ultrasonic echocardiography, family history of CHD, history of peripheral vascular diseases, renal failure, left main disease, heart failure or atrial fibrillation. However, patients with MetS without diabetes tended to be female $(21.2 \%$ vs. $19.7 \%$ and $11.3 \%, P=0.0002)$, have stable angina pectoris $(7.66$ vs. $5.26 \%$ and $3.11 \%, P<0.0001)$. Patients with MetS and diabetes had higher body mass index (BMI) $(33 \pm 24.8)$, were more likely to have unstable angina pectoris $(22.4 \%$ 
Table 1 Baseline characteristics of the patients

\begin{tabular}{|c|c|c|c|c|c|}
\hline Variables & MetS without DM N = 222 & MetS with DM N = 76 & No MetS $N=868$ & $P$ Value & $P$ Value of Fisher Test \\
\hline Age $(y r)$ & $59.97 \pm 8.22$ & $58.08 \pm 7.72$ & $58.95 \pm 9.48$ & 0.2052 & \\
\hline BMI $\left(\mathrm{kg} / \mathrm{m}^{2}\right)$ & $32.89 \pm 22.22$ & $32.96 \pm 24,77$ & $24.19 \pm 2.26$ & $<0.0001$ & \\
\hline Smoking & $131(59.01 \%)$ & $47(61.84 \%)$ & 489 (56.34\%) & 0.5406 & \\
\hline Creatinine (tjmol/L) & $103.42 \pm 110.68$ & $133.75 \pm 196.34$ & $110.38 \pm 123.25$ & 0.5821 & \\
\hline CPU time (min) & $58.88 \pm 66.51$ & $63.88 \pm 64.48$ & $58.48 \pm 76.78$ & 0.8309 & \\
\hline ACC Time (min) & $36.89 \pm 42.16$ & $41.37 \pm 42.53$ & $36.39 \pm 40.29$ & 0.5945 & \\
\hline Diseased coronary artery & $2.77 \pm 0.50$ & $2.72 \pm 0.48$ & 2.7110 .57 & 0.2480 & \\
\hline LVEF & $59.73+9.34$ & $58.41+8.36$ & $59.15+10.04$ & 0.5599 & \\
\hline Preoperative intravenous use of nitrates & $0.06 \pm 0.24$ & $0.13 \pm 0.34$ & 0.0510 .21 & 0.0096 & \\
\hline Female & $47(21.17 \%)$ & $15(19.74 \%)$ & $98(11.29 \%)$ & 0.0002 & \\
\hline Hypertension & $222(100 \%)$ & $76(100 \%)$ & 0 & $<0.0001$ & \\
\hline Hyperlipidemia & $222(100 \%)$ & $76(100 \%)$ & 0 & $<0.0001$ & \\
\hline Cerebrovascular events & $13(5.86 \%)$ & $6(7.89 \%)$ & $22(2.53 \%)$ & 0.0057 & \\
\hline Renal failure & 0 & 0 & $2(0.23 \%)$ & 0.7090 & 1.0000 \\
\hline Elevated fasting glucose & 0 & $76(100 \%)$ & 0 & $<0.0001$ & \\
\hline Thrombolytic therapy & $14(6.31 \%)$ & $4(5.26 \%)$ & $112(12.90 \%)$ & 0.0050 & \\
\hline PCl history & 0 & 0 & 0 & & \\
\hline \multicolumn{6}{|l|}{ Angina pectoris } \\
\hline Unstable angina pectoris & $34(15.32 \%)$ & $17(22.37 \%)$ & $59(6.80 \%)$ & $<0.0001$ & \\
\hline Stable angina pectoris & $17(7.66 \%)$ & $4(5.26 \%)$ & $27(3.11 \%)$ & $<0.0001$ & \\
\hline Myocardial infarction & $90(40.54 \%)$ & $38(50.00 \%)$ & $451(51.96 \%)$ & 0.0099 & \\
\hline Family history & $21(9.46 \%)$ & $5(6.58 \%)$ & $54(6.22 \%)$ & 0.2333 & \\
\hline Left main disease & $55(24.77 \%)$ & $20(26.32 \%)$ & $270(31.11 \%)$ & 0.1481 & \\
\hline Peripheral artery disease & $21(9.46 \%)$ & $4(5.26 \%)$ & $63(7.26 \%)$ & 0.3994 & \\
\hline Heart failure & $2(0.90 \%)$ & $2(2.63 \%)$ & $18(2.07 \%)$ & 0.4590 & 0.4242 \\
\hline Atrial fibrillation & $6(2.70 \%)$ & $3(3.95 \%)$ & $15(1.73 \%)$ & 0.3211 & 0.2247 \\
\hline
\end{tabular}

vs. $15.3 \%$ and $6.80 \%, P<0.0001$ ), use intravenous nitrates $(0.13 \pm 0.34)$, and have a more extensive history of cerebrovascular events $(7.89 \%$ vs. $5.86 \%$ and $2.53 \%, P=$ 0.0057). Control group members were more likely to have thrombolytic therapy $(12.9 \%$ vs. $6.31 \%, 12.9 \% . P=$ $0.0050)$ and have a history of myocardial infarction (52\% vs. $40.5 \%, 50.0 \% . P=0.0099)$.

\section{Perioperative (short-term) outcomes}

Table 2 helps illustrate that there were no significant differences in ICU stay, ventilation time, in-hospital postoperative complications, epinephrine use, stroke, atrial fibrillation, renal failure, coma, myocardial infarction and repeated revascularization among the groups. The groups did differ significantly in perioperative MACCE (30.3\% vs. $21.2 \%$ and $16.7 \%, P=0.0071)$ and mortality $(11.8 \%$ vs. $2.7 \%$ and $3.11 \%, P=0.0003)$. As the Chi-square and Fisher's Exact test results in Table 3 show, the MetS with diabetes group had higher rates of perioperative mortality than Mets without diabetes $(P=$
0.0017, P of Fisher Test $=0.0039$ ), while rates of MACCE were equal. No significant differences in mortality or MACCE were found between the Mets without diabetes and control groups.

\section{Long-term follow-up for mortality}

The median follow up duration was 59.3 months. Compared to non-diabetetic MetS group, patients with MetS and diabetes suffered significantly greater long-term mortality (HR: 3.048; 95\%CI: 1.022-9.086; $P=0.0456$ ). There was no significant difference between the nondiabetic MetS and control groups (Table 4).The KaplanMeier curves in Fig. 2 illustrate the trend of long-term mortality among three groups. MetS with diabetes group showed significant higher mortality than control group during the annual follow-up while Mets without diabetes group showed no significant difference. (Logrank test $P=0.0075)$. Multivariable Cox regression model was used to adjust the confounding factors and analyze the association among MetS, diabetes and 
Table 2 Postoperative characteristics

\begin{tabular}{llllll}
\hline Variables & MetS without DM N=222 & MetS with DM N=76 & No MetS N=868 & $P$ Value & $P$ Value of Fisher Test \\
\hline ICU stay(h) & $60.33 \pm 67.79$ & $48.26 \pm 38.29$ & $61.06 \pm 63.92$ & 0.2400 \\
Ventilation Time $(\mathrm{h})$ & $18.23 \pm 22.63$ & $16.22 \pm 7.12$ & $15.86 \pm 24.17$ & 0.3938 \\
Postoperative Complications & 0 & 0 & $7(0.81 \%)$ & 0.2985 \\
Epinephrine Use & $28(12.61 \%)$ & $14(18.42 \%)$ & $93(10.71 \%)$ & 0.1141 & 0.5986 \\
Stroke & $1(0.45 \%)$ & $1(1.32 \%)$ & $4(0.46 \%)$ & 0.6005 & 0.6846 \\
Atrial Fibrillation & $13(5.86 \%)$ & $4(5.26 \%)$ & $62(7.14 \%)$ & $1(0.12 \%)$ & 0.1151 \\
Renal Failure & $1(0.45 \%)$ & $1(1.32 \%)$ & $7(0.81 \%)$ & 0.7214 & 0.4018 \\
Coma & $2(0.90 \%)$ & 0 & $8(0.92 \%)$ & 0.3693 & 0.0815 \\
Myocardial Infarction & $3(1.35 \%)$ & $2(2.63 \%)$ & $33(3.80 \%)$ & 0.5232 \\
Repeated Revascularization & $5(2.25 \%)$ & $3(3.95 \%)$ & $27(3.11 \%)$ & 0.0003 \\
Mortality & $6(2.70 \%)$ & $9(11.84 \%)$ & $145(16.71 \%)$ & 0.0071 \\
MACCE & $47(21.17 \%)$ & $23(30.26 \%)$ & & 0.2403 \\
\hline
\end{tabular}

mortality. As shown in Table 5, patients in MetS with diabetes group had increased long-term follow-up mortality (adjusted HR: 4.299; 95\% CI: 1.392-13.277; $P=0.0113$ ). In addition, old age (adjusted HR: $1.061 ; 95 \%$ CI: $1.021-1.103 ; P=0.0027$ ) and smoking (adjusted HR: 2.103; 95\%CI: 1.0194.341; $P=0.0443$ ) were also risk factors for the long-term mortality.

\section{Long-term follow-up for MACCE}

The univariate Logistic regression model showed increse in long-term MACCE in the MetS with diabetes compared to non-diabetes MetS groups (Table 6). Patients in the Control group had a lower incidence of MACCE compared to the non-diabetes MetS group (OR: 0.741; 95\% CI: 0.601-0.913; $P=$ 0.0050). Multivariable Logistic regression adjusted the confounding factors to analyze long-term MACCE. As in Table 7, patients in MetS with diabetes group showed increasing rates of MACCE (adjusted OR: 1.674; 95\% CI: $1.004-2.792 ; P=0.0484)$. Patients in control group had decreasing rate of MACCE (Adjusted OR: 0.792; 95\% CI: 0.639-0.981; $P=0.0329)$. In addition, old age (Adjusted OR: 1.029; 95\% CI: 1.0171.041; $P<0.0001$ ), cerebrovascular events (Adjusted OR: $1.964 ; 95 \%$ CI: $1.395-2.767 ; P=0.0001)$ and aortic cross-clamp time (Adjusted OR: 1.005; 95\% CI: $1.003-1.007 ; P<0.0001)$ are also risk factors for the rate of MACCE.

\section{Discussion}

In the present study, differences of both short and long term mortality were found between diabetic MetS patients and non-diabetic MetS patients, while were not found between non-diabetic MetS patients and nonMetS paients, which indicated that diabetes played an important role in the death of MetS patients. We also found that, compared with non-diabetic MetS patients, diabetic MetS patients had higher rates of MACCE, and so did non-diabetic MetS patients when compared with control patients. So both MetS and diabetes may contributes to the development of MACCE. Besides, age, smoking, cerebralvascular events and aortic cross-clamp time showed in the substudies were also the risk factors of mortality or morbidity after CABG.

There's close contact between atherosclerosis, Metabolic Syndrome and diabetes. A conception called "Cardiometabolic Diseases" revolve around a complicated cluster of events including visceral adiposity, MetS, type 2 diabetes and CHD [20]. Insulin resistance is the major contributors to the pathological basis of MetS and Diabetes (which may contains confounding factors). Glucolipotoxicity and chronic inflammatory state generated under insulin resistance lead to dysfunction of VEC. On the other hand, pancreatic dysfunction from islet VEC leads to low perfusion of pancreas islet and aggravates the decompensation. In addition, one study has shown that diabetes tends to leave patients in a hypercoagulable state [21]. These abnormalities work

Table 3 MetS and Perioperative (Short-term) Mortality and MACCE

\begin{tabular}{|c|c|c|c|c|}
\hline \multirow[t]{2}{*}{ Variables } & \multicolumn{2}{|c|}{ Mortality } & \multicolumn{2}{|l|}{ MACCE } \\
\hline & $P$ Value & $P$ Value of Fisher Test & $P$ Value & $P$ Value of Fisher Test \\
\hline MetS with DM vs. MetS without DM & 0.0017 & 0.0039 & 0.1066 & 0.1181 \\
\hline No MetS vs. MetS without DM & 0.1002 & 1.0000 & 0.1190 & 0.1382 \\
\hline MetS with DM vs. No MetS & 0.0001 & 0.0014 & 0.0030 & 0.0048 \\
\hline
\end{tabular}


Table 4 MetS and Long-term Mortality

\begin{tabular}{|c|c|c|c|c|c|c|}
\hline \multirow[t]{2}{*}{ Variables } & \multicolumn{4}{|c|}{ Un-adjusted } & \multicolumn{2}{|l|}{ Adjusted } \\
\hline & $\mathrm{HR}$ & $95 \% \mathrm{Cl}$ of $\mathrm{HR}$ & $P$ Value & $H R$ & $95 \% \mathrm{Cl}$ of $\mathrm{HR}$ & $P$ Value \\
\hline MetS with DM vs. MetS without DMs & 3.048 & $1.022-9.086$ & 0.0456 & 4.299 & $1.392-13.277$ & 0.0113 \\
\hline No MetS vs. MetS without DM & 0.862 & $0.354-2.096$ & 0.7426 & 0.992 & $0.388-2.540$ & 0.9870 \\
\hline
\end{tabular}

together in developing atherosclerosis. Complex coronary lesions, such as bifurcation and ostial lesions, are significantly more common in diabetic patients [22].

In cardiovascular surgery, $\mathrm{CPB}$, hypothermic anesthesia and blood dilution trigger intense stress reactions, which are characterized by hyperglycemia and hyperinsulinemia. And the ischemia-reperfusion injury of myocardium reduces the reaction of myocardial cells to insulin, i.e., myocardial insulin resistance. A further study found that patients with blood glucose values $>200 \mathrm{mg} / \mathrm{dl}$ immediately after CABG had an increased risk of complications, including mortality [9]. Another study reported chronic hyperglycemia is associated with acute kidney injury after CABG [23].

MetS and diabetes are becoming increasingly common as the result of worldwide change in diet and lack of exercise, leading to enormous economic burdens on society [24-26]. CVD already is the most common cause of death in China. Determining the impacts of MetS and diabetes on outcomes after CABG will help to manage operative risk for a growing proportion of the population. Furthermore, it is crucial for the development of public health policy and clinical guidelines for prevention and treatment which may lead to a controlled form of MetS and diabetes, potentially improving long-term outcomes after CABG.

But very few studies have previously attempted to delineate the role of MetS and/or diabetes on the

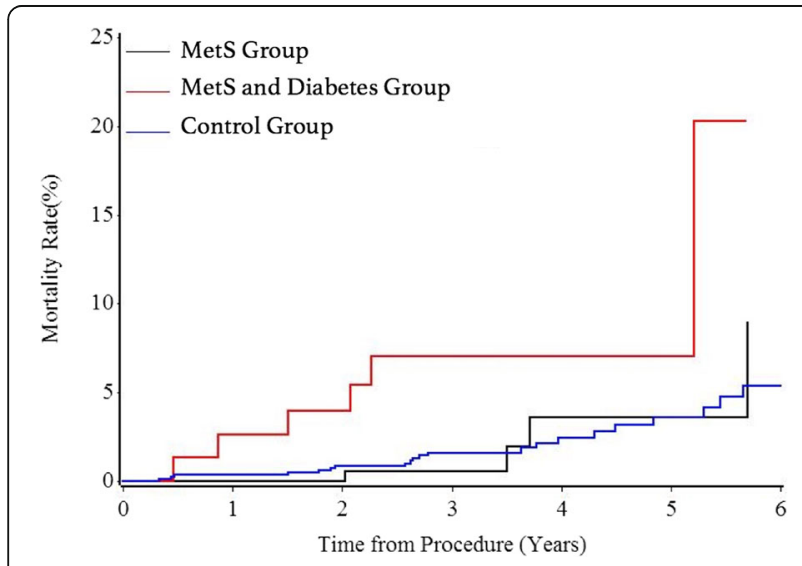

Fig. 2 Kaplan-meier for mortality rate following-up 5 years. MetS with diabetes group showed significant higher mortality than control group during the annual follow-up while Mets without diabetes group showed no significant difference. (Log-rank test $P=0.0075$ ) immediate and long-term MACCE and mortality. This study has filled in the blank to some extent.

Studies have found contradictory results about the effect of MetS on outcomes after CABG. In the mainstream view, MetS predicts outcomes after CABG $[4,6,7,10]$. Recently contributors propose that MetS has no detrimental effect on either the pre-operative risk factors or the outcome after CABG [27, 28]. Further experiments are necessary to solve the mystery.

Contradictory results exist on the effect of diabetes on outcomes after CABG. Combining 146,000 patients from various hospitals showed a mortality of $3.7 \%$ for patients with diabetes mellitus and $2.7 \%$ for those without diabetes mellitus [29]. A propensity-matched study containing 1122 subjects showed that long-term survival is significantly lower in patients with diabetes compared to non-diabetics after CABG [30]. A survival analysis of 910 CABG patients by the Life Table method found that patients without diabetes had at least equal survival in 16 years after CABG compared to their matched background populations. While survival of DM patients started to decline few years after the operation [31]. But in these studies, DM or non-DM suffered the influence of MetS, which may show more strongly in DM group.

There are also researches involvles both MetS and diabetes attempting to find out their influnces on the outcomes of CABG [32]. In a 15-year observational study MetS increased all-cause and cardiac mortality of non-diabetic patients (hazard ratio 1.34, $P=0.028$ and $2.31, P=0.002$, respectively) while no increased mortality

Table 5 Multivariable-adjusted hazard ratio of long-term mortality

\begin{tabular}{llll}
\hline Variables & $P$-Value & $\mathrm{HR}$ & $\mathrm{HR9S} \% \mathrm{Cl}$ \\
\hline MetS with DM & 0.0113 & 4.299 & $1.392-13.277$ \\
Non MetS & 0.9870 & 0.992 & $0.388-2.540$ \\
Age & 0.0027 & 1.061 & $1.021-1.103$ \\
Aortic Cross-clamp Time & 0.3390 & 0.996 & $0.988-1.004$ \\
Sex & 0.8026 & 0.867 & $0.282-2.660$ \\
Smoking & 0.0443 & 2.103 & $1.019-4.341$ \\
Cerebrovascular Events & 0.2245 & 2.143 & $0.626-7.331$ \\
Peripheral Artery Disease & 0.0904 & 2.537 & $0.864-7.450$ \\
Thrombolytic Therapy & 0.3519 & 1.535 & $0.623-3.787$ \\
Myocardial Infarction & 0.5509 & 0.812 & $0.410-1.609$ \\
Left Main Disease & 0.9144 & 1.038 & $0.528-2.040$ \\
\hline
\end{tabular}


Table 6 MetS and MACCE in the 5-year follow-up

\begin{tabular}{llllllll}
\hline Variables & \multicolumn{2}{l}{ Un-adjusted } & & & \multicolumn{2}{c}{ Adjusted } \\
\cline { 2 - 4 } & OR & $95 \%$ Cl of OR & $P$ Value & OR & & $95 \%$ Cl of OR & $P$ Value \\
\hline MetS with DM vs. MetS without DM & 1.603 & $0.971-2.647$ & 0.0652 & 1.674 & $1.004-2.792$ & 0.0484 \\
No MetS vs. MetS without DM & 0.741 & $0.601-0.913$ & 0.0050 & 0.792 & $0.639-0.981$ & 0.0329 \\
\hline
\end{tabular}

was found among diabetic patients [4]. The result of a retrospective reseach showed the motality of diabetic patients with no MetS after CABG had no difference with patients with neither diabetes nor MetS, however, patients with both diabetes and MetS were found out worse than diabetic patients without MetS which conflict with the former [2]. And in the same research, the risk of mortality was increased by 2.69 -fold (95\% CI 1.43 to $5.06 ; p=0.002)$ in patients with MS and diabetes and 2.36 -fold (95\% CI 1.26 to $4.41 ; p=0.007$ ) in patients with MS and no diabetes compared with no diabetes and no MetS patients while there's no comparison between the two cohort. In this study, we found that patients with MetS plus diabetes had higher rates of postoperative and long-term mortality and MACCE compared with MetS patients without diabetes. However, patients with diabetes and other 2 criteria of MetS might be included in MetS with diabetes in the two researches mentioned above but excluded in current study. Besides, difference of the diagnostic criteria of MetS and statistical methods adapted in different articles may also have influences to the conclusion. In the future, it's of meaning to take non-diabetic 3- factors MetS, non-MetS diabetes and diabetes plus 3-factors MetS or nondiabetic 3- factors MetS and 2- factors MetS plus diabetes into comparison to find out the further role of MetS or diabetes in the outcome of CABG. Based on which, a risk stratification used to conduct preoperative preparation or even the whole perioperative management maybe possible.

Table 7 Multivariable-Adjusted Odd Ratio of Long-term MACCE

\begin{tabular}{llll}
\hline Variables & P-Value & OR & OR 95\% Cl \\
\hline MetS with DM & 0.0484 & 1.674 & $1.004-2.792$ \\
Non MetS & 0.0329 & 0.792 & $0.639-0.981$ \\
Age & $<.0001$ & 1.029 & $1.017-1.041$ \\
Aortic Cross-clamp Time & $<.0001$ & 1.005 & $1.003-1.007$ \\
Sex & 0.8247 & 0.969 & $0.732-1.283$ \\
Smoking & 0.0704 & 1.207 & $0.984-1.480$ \\
Cerebrovascular Events & 0.0001 & 1.964 & $1.395-2.767$ \\
Peripheral Artery Disease & 0.9246 & 0.983 & $0.696-1.389$ \\
Thrombolytic Therapy & 0.1203 & 1.286 & $0.936-1.766$ \\
Myocardial Infarction & 0.3447 & 1.100 & $0.903-1.339$ \\
Left Main Disease & 0.1434 & 0.857 & $0.697-1.054$ \\
\hline
\end{tabular}

\section{Limitations}

This was a retrospective study at a single center among a cohort of exclusively Chinese patients. Bias may remain despite multivariate adjustments to reduce overt sources. Also, MetS was assessed using BMI because waist circumferences were not available. Although international guidelines suggest the use of waist circumference to classify obesity, several studies have demonstrated that there is no significant difference between the two classification methods, i.e., BMI or waist circumference.

\section{Conclusions}

There were significance differences between the three groups in perioperative Major Adverse Cerebral Cardiovascular Events and mortality after Coronary Artery Bypass Graft. Compared with non-diabetic Metabolic Syndrome patients, patients with Metabolic Syndrome and diabetes had higher long-term Major Adverse Cerebral Cardiovascular Events and mortality. While patients free of MetS and diabetes were associated with lower incidence of long-term Major Adverse Cerebral Cardiovascular Events after Coronary Artery Bypass Graft.

\section{Abbreviations}

AGE: Advanced glycosylation end products; BMl: Body mass index; CABG: Coronary artery bypass graft; CHD: Coronary heart disease; CNS: Central nervous system; CPB: Cardiopulmonary bypass; DM: Diabetes mellitus; EF: Ejection fraction; MACCE: Major adverse cerebral cardiovascular events; MetS: Metabolic syndrome; MI: Myocardial infarction; NIDDM: Non insulin dependent diabetes mellitus; VEC: Vascular endothelial cells

\section{Acknowledgements}

We would like to express our great appreciation to Pro. Hushan Ao for their valuable and constructive suggestions during the planning and development of this research work. We also would like to express special thanks to Mr. Sipeng Chen for their critical suggestions on data collection and data analysis.

Funding

Not applicable.

Availability of data and materials

All data generated or analyzed during this study are included in this published article and Fuwai dataset.

Authors' contributions

LW: Study design, patient recruitment, and data collection and writing up the first draft; XQ: Study design, patient recruitment, and data collection; XT: Data analysis, data interpretation; MW: Critical revision, final approval of publication; HA: Study conception and design, critical revision, final approval of publication. All authors read and approved the final manuscript. 


\section{Ethics approval and consent to participate}

The study was conducted in accord with the Declaration of Helsinki principals and approved by the Fuwai Hospital Institutional Review Board. Informed consent (Consent to Participate) was obtained from each of the patients.

\section{Consent for publication}

Informed consent was obtained from the patient for publication of this report and available for review.

\section{Competing interests}

The authors declare that they have no competing interests.

\section{Publisher's Note}

Springer Nature remains neutral with regard to jurisdictional claims in published maps and institutional affiliations.

\begin{abstract}
Author details
'Department of Anesthesiology, Beijing Fuwai Hospital, National Center for Cardiovascular Diseases, Chinese Academy of Medical Sciences and Peking Union Medical College, No. 167, Beilishi Road, West District of Beijing, Beijing 100037, China. ${ }^{2}$ Department of Cardiac Surgery, Beijing Fuwai Hospital, National Center for Cardiovascular Diseases, Chinese Academy of Medical Sciences and Peking Union Medical College, Beijing, China.
\end{abstract}

Received: 14 July 2017 Accepted: 5 December 2017

Published online: 04 January 2018

\section{References}

1. Grundy SM, Cleeman JI, Daniels SR, Donato KA, et al. Diagnosis and management of the metabolic syndrome: an American Heart Association/ National Heart, Lung, and Blood Institute scientific statement. Curr Opin Cardiol. 2006;21(1):1-6. PMID: 16355022

2. Echahidi N, Pibarot P, Despres JP, Daigle JM, et al. Metabolic syndrome increases operative mortality in patients undergoing coronary artery bypass grafting surgery. J Am Coll Cardiol. 2007:50(9):843-51. PMID: 17719470

3. Moebus S, Balijepalli C, Lösch C, Göres L, et al. Age- and sex-specific prevalence and ten-year risk for cardiovascular disease of all 16 risk factor combinations of the metabolic syndrome - a cross-sectional study. Cardiovasc Diabetol. 2010;9:34. PMID: 20696055

4. Kajimoto K, Kasai T, Miyauchi K, Hirose H, et al. Metabolic syndrome predicts 10-year mortality in non-diabetic patients following coronary artery bypass surgery. Circ J. 2008;72(9):1481-6. PMID: 18724026

5. Taggart DP. Impact of type 1 and 2 Diabetes Mellitus on long-term outcomes after CABG. J Am Coll Cardiol. 2015;65(16):1653-4. PMID: 25908070

6. Isomaa B. A major health hazard: the metabolic syndrome. Life Sci. 2003; 73(19):2395-411. PMID: 12954449

7. Angeloni E, Melina G, Benedetto U, Refice S, et al. Metabolic syndrome affects midterm outcome after coronary artery bypass grafting. Ann Thorac Surg. 2012;93(2):537-44. PMID: 22197615

8. Koshizaka M, Lopes RD, Schulte PJ, Hafley GE, et al. Long-term clinical and angiographic outcomes in patients with diabetes undergoing coronary artery bypass graft surgery: results from the project of ex-vivo vein graft engineering via Transfection IV trial. Am Heart J. 2015;169(1):175-84. PMID: 25497264

9. Jones KW, Cain AS, Mitchell JH, Millar RC, et al. Hyperglycemia predicts mortality after $C A B G$ : postoperative hyperglycemia predicts dramatic increases in mortality after coronary artery bypass graft surgery. J Diabetes Complicat. 2008;22(6):365-70. PMID: 18413193

10. Ardeshiri M, Faritus Z, Bakhshandeh $\mathrm{H}$, Kargar F, et al. Impact of metabolic syndrome on mortality and morbidity after coronary artery bypass grafting surgery. Res Cardiovasc Med. 2014;3(3):e20270. PMID: 25478548

11. Expert Panel on Detection, Evaluation, and Treatment of High Blood Cholesterol in Adults. Executive summary of the third report of the National Cholesterol Education Program (NCEP) expert panel on detection, evaluation, and treatment of high blood cholesterol in adults (adult treatment panel III). JAMA. 2001;285(19):2486-97. PMID: 11368702

12. Wong ND, Sciammarella MG, Polk D, Gallagher A, et al. The metabolic syndrome, diabetes, and subclinical atherosclerosis assessed by coronary calcium. J Am Coll Cardiol. 2003;41(9):1547-53. PMID: 12742296
13. Janssen I, Katzmarzyk PT, Ross R. Body mass index, waist circumference, and health risk: evidence in support of current National Institutes of Health guidelines. Arch Intern Med. 2002;162(18):2074-9. PMID: 12374515

14. Wang $X$, Zheng $Z$, Ao H, Zhang S, et al. Effects of aprotinin on short-term and long-term outcomes after coronary artery bypass grafting surgery. Ann Thorac Surg. 2010;89(5):1489-95. PMID: 20417766

15. Mangano DT. Perioperative cardiac morbidity. Anesthesiology. 1990;72(1): 153-84. PMID: 2404426

16. Cao L, Young N, Liu H, Silvestry S, et al. Preoperative aspirin use and outcomes in cardiac surgery patients. Ann Surg. 2012;255(2):399-404. PMID: 21997805

17. Mangano DT, Browner WS, Hollenberg M, London MJ, et al. Association of perioperative myocardial ischemia with cardiac morbidity and mortality in men undergoing noncardiac surgery. The study of Perioperative ischemia research group. N Engl J Med. 1990;323(26):1781-8. PMID: 2247116

18. Calafiore AM, Di Mauro M, Di Giammarco G, Contini M, et al. Effect of diabetes on early and late survival after isolated first coronary bypass surgery in multivessel disease. J Thorac Cardiovasc Surg. 2003;125(1):144-54. PMID: 12538998

19. Munnee K, Bundhun PK, Quan H, Tang Z, et al. Comparing the clinical outcomes between insulin-treated and non-insulin-treated patients with type 2 diabetes mellitus after coronary artery bypass surgery: a systematic review and meta-analysis. Medicine (Baltimore). 2016;95(10):e3006. PMID: 26962814

20. Westergren HU, Svedlund S, Momo RA, Blomster JI, et al. Insulin resistance, endothelial function, angiogenic factors and clinical outcome in nondiabetic patients with chest pain without myocardial perfusion defects. Cardiovasc Diabetol. 2016;15:36. PMID: 26892461

21. Pomero F, Di Minno MN, Fenoglio L, Gianni M, et al. Is diabetes a hypercoagulable state? A critical appraisal. Acta Diabetol. 2015;52(6):1007-16 PMID: 25850539

22. Baris N, Akdeniz B, Uyar S, Ozel E, et al. Are complex coronary lesions more frequent in patients with diabetes mellitus? Can J Cardiol. 2006;22(11):9357. PMID: 16971978

23. Oezkur M, Wagner M, Weismann D, Krannich JH, et al. Chronic hyperglycemia is associated with acute kidney injury in patients undergoing CABG surgery-a cohort study. BMC Cardiovasc Disord. 2015;15:41. PMID: 25964053

24. Lakka HM, Laaksonen DE, Lakka TA, Niskanen LK, et al. The metabolic syndrome and total and cardiovascular disease mortality in middle-aged men. JAMA. 2002;288(21):2709-16. PMID: 12460094

25. Krauss RM, Winston M, Fletcher BJ, Grundy SM. Obesity : impact on cardiovascular disease. Circulation. 1998;98(14):1472-6. PMID: 9760304

26. Meigs JB. Epidemiology of the metabolic syndrome. Am J Manag Care. 2002:8(11 Suppl):S283-92. quiz S293-6. PMID: 12240700

27. Swart MJ, De Jager WH, Kemp JT, Nel PJ, et al. The effect of the metabolic syndrome on the risk and outcome of coronary artery bypass graft surgery. Cardiovasc J Afr. 2012;23(7):400-4. PMID: 22914999

28. Tie HT, Shi $R$, Zhang $M$, Zhang $C$, et al. Risk of major adverse cardiovascular events in patients with metabolic syndrome after revascularization: a metaanalysis of eighteen cohorts with 18,457 patients. Metabolism. 2015;64(10): 1224-34. PMID: 26231436

29. Carson JL, Scholz PM, Chen AY, Peterson ED, Gold J, Schneider SH. Diabetes mellitus increases short-term mortality and morbidity in patients undergoing coronary artery bypass graft surgery. J Am Coll Cardiol. 2002;40: 418-23. PMID: 12142105

30. Pang $P Y$, Lim YP, Ong KK, Chua YL, et al. Long-term prognosis in patients with diabetes mellitus after coronary artery bypass grafting: a propensitymatched study. Ann Acad Med Singap. 2016;45(3):83-90. PMID: 27146460

31. Hällberg V, Palomäki A, Lahtela J, Voutilainen S, et al. Associations of metabolic syndrome and diabetes mellitus with 16-year survival after CABG. Cardiovasc Diabetol. 2014;13:25. PMID: 24447406

32. Tzimas P, Petrou A, Laou E, Papadopoulos G, et al. Impact of metabolic syndrome in surgical patients: should we bother? Br J Anaesth. 2015;115(2): 194-202. PMID. 26109210 